Article

\title{
Ionospheric Science: An Example of the Importance of Diversity in Approaches to Scientific Research
}

\author{
Mike Hapgood (D)
}

check for updates

Citation: Hapgood, M. Ionospheric Science: An Example of the Importance of Diversity in Approaches to Scientific Research. Atmosphere 2022, 13, 394. https:// doi.org/10.3390/atmos13030394

Academic Editors: Ljiljana R. Cander and Bruno Zolesi

Received: 15 February 2022

Accepted: 24 February 2022

Published: 27 February 2022

Publisher's Note: MDPI stays neutral with regard to jurisdictional claims in published maps and institutional affiliations.

Copyright: (C) 2022 by the author. Licensee MDPI, Basel, Switzerland. This article is an open access article distributed under the terms and conditions of the Creative Commons Attribution (CC BY) license (https:// creativecommons.org/licenses/by/ $4.0 /)$.
RAL Space, STFC Rutherford Appleton Laboratory, Harwell Campus, Didcot OX11 0QX, Oxfordshire, UK; mike.hapgood@stfc.ac.uk

\begin{abstract}
This paper discusses the strategic importance of contemporary ionospheric science. It outlines some key features of the evolution of the science from the first practical experiments in the 1920s through to the diverse inter-disciplinary science of today. This science includes fundamental studies of partially ionised plasmas and of the complex systems that arise when those plasmas are coupled to neutral atmospheres and magnetospheres. However, the science also has great potential to deliver societal benefits if the science can be refined to obtain a deep physical understanding of ionospheric phenomena and that understanding is then transitioned into use by operational services such as forecasts of ionospheric conditions. Thus, ionospheric science is now very similar in form to other environment sciences and, the same as them, needs to be positioned in a diverse scientific culture that supports the full range of science research, including not only curiosity-driven studies, but also targeted research to deepen our physical understanding to a level that is sufficient to enable a transition to operational services. That diversity also includes support for that transition and also facilitates feedback from operations teams to researchers. Such feedback can be a powerful stimulus for future research.
\end{abstract}

Keywords: ionosphere; partially ionised plasmas; discovery science; curiosity-driven science; targeted science; research-to-operations; ionospheric propagation; trans-ionospheric radio systems; funding policy

\section{Introduction}

Ionospheric science has a long history of achievement going back almost a century. It has its practical roots in the 1920s when the first wave of great developments in electronics, in particular the triode valve, enabled radio measurements of conditions in Earth's upper atmosphere. Its evolution in the following decades was often linked to astronomy and space science as the exploration of the upper atmosphere was then considered part of astronomy, for example being discussed in popular astronomy textbooks published in Mid-20th Century, e.g., see [1].

However, in the later decades of the 20th Century, those links became more diffuse as ionospheric science matured and became more inter-disciplinary. For example, the polar ionosphere became a major focus from the 1970s onwards, reflecting both (a) the growing scientific interest in the coupling between the ionosphere and the magnetosphere and (b) the improvements in Arctic and Antarctic transport, communications, and energy infrastructures that facilitated the deployment of advanced instruments in those regions, e.g., the European Incoherent Scatter (EISCAT) radars first deployed in Northern Scandinavia [2] and later in Svalbard [3]. Another, very different, example was the application of ionospheric science to study radio propagation through the ionosphere. Originally focused on high-frequency (HF) radio communications, this expanded greatly from the 1960s onwards with the development of radio-frequency systems for a wide range of satellite and groundbased applications [4,5]. A further example, becoming more significant towards the end of the 20th Century, was the scientific interest in coupling between the lower atmosphere 
and the ionosphere. This "vertical coupling" is now recognised as an important source of day-to-day variability in the ionosphere and is a major research topic that links ionospheric science to atmospheric science. Its importance to modern science may be summed up in a phrase from the late Georg Witt [6], who, at a 2008 workshop, emphasised to us all that "The atmosphere is one system".

This evolution of ionospheric studies into an interdisciplinary science, with strong links both to other science disciplines and to radio system engineering, has led to a wealth of excellence, both in research and in the application of that research to the benefit of societies around the world. However, it has also led to misunderstandings in other parts of the scientific community where it has been considered that it is now time for ionospheric, and other space weather studies to be focused on applications, e.g., see the recent discussion by Lugaz et al. [7]. This paper addresses that misunderstanding. The paper has three main sections. Section 2 sets the scene by outlining how ionospheric science developed in the early 20th Century and also developed links with both the applications community and the astronomy community. This is followed in Section 3 by a short review of some current key issues in ionospheric science showing how it has matured into a highly interdisciplinary topic that is best considered as a branch of the environmental sciences. Section 4 then shows how, as with other environment sciences, ionospheric science needs to be positioned in a scientific culture that supports a diversity of approaches to research, a mix of curiosity-driven studies to address the fundamental understanding of a complex natural system, targeted research to develop a deep understanding of environmental conditions that have profound impacts on human societies, and effective mechanisms to support the transition of research outputs into operational services that help to manage our responses to those conditions. These three sections are followed by a summary that highlights the key conclusions of the paper.

\section{Historical Background}

Modern ionospheric science has advanced greatly since the first suggestion of the existence of the ionosphere by Heaviside in 1902 [8] and the pioneering work of key figures such as Appleton (e.g., see [9]) and Breit and Tuve (e.g., see [10]). It advanced greatly in the Mid-Twentieth Century through both theoretical advances such as Bate's work on dissociative recombination [11] and the wider understanding of the upper atmosphere that came from early satellite missions. Those advances continued through to the end of that century and into the new one, with many advances in understanding ionospheric storms and irregularities, and in particular much focus on the high-latitude ionosphere.

As noted above, these scientific advances have always had a strong link with the radio applications community because of the practical importance of trans-ionospheric radio links whose propagation varies with the conditions in the ionosphere. This first came to prominence in the 1920s as high-frequency (HF) radio became the primary means of longdistance radio communications [12]. HF superseded the low-frequency systems (typically a few 10s of kHz) deployed earlier in the 20th Century, e.g., the Franco-American BordeauxLafayette system, which operated at $15 \mathrm{kHz}$ [13]. HF provided much more bandwidth, thereby facilitating more sophisticated services, not the least of which was human voice rather than Morse code. However, it was quickly recognised that HF signals could be severely disturbed by space weather events such as magnetic storms [14,15] and solar flares [16]. Thus, ionospheric science was from the outset a topic where there was a strong link between the applications community and wider research into the behaviour of the Sun and its impact on Earth. Many leading astronomers welcomed ionospheric observations as a complement to other methods of studying the Sun. For example, Harold Newton, the leading solar astronomer at Greenwich Observatory in the UK [17-21], exploited several mid-latitude ionospheric effects that are indicators of solar flares: both HF blackout that occurs when solar X-rays enhance the $\mathrm{D}$ region and the magnetic crochets that occur when the solar Lyman-alpha enhances E region conductivity. 
Thus, from the 1930s onwards, ionospheric science was seen as an area where there was synergy between research and applications and that there was a need for operational capabilities that could monitor and forecast ionospheric conditions. For example, whilst telephone services between the UK and USA were first introduced in 1927 using lowfrequency $(60 \mathrm{kHz})$ links, they were quickly complemented in the following year by services using HF links [22]. However, these links were subject to disruption during intense geomagnetic storms such as that in January 1938 [23,24]. Ionospheric studies became very important during the Second World War with all participants using HF as a key communications technique wherever and whenever land lines were not available. Thus, they continued ionospheric research together with operational monitoring, sometimes making great efforts to deploy monitoring instruments in strategic locations, e.g., the British deployment of ionosondes on the harsh and disputed islands of Svalbard in the High Arctic [25].

After that war, HF continued to be a mainstay of long-distance communications, but was gradually complemented by other systems. For example, the 1950s and 1960s saw extensive deployment of undersea coaxial cables capable of carrying multiple telephone circuits, thus removing a vulnerability to ionospheric effects (this technology was in turn superseded by the undersea optical fibre cables that now carry $99 \%$ of transoceanic telephone and Internet communications). Another example of technological progress has been the development of satellite communications from the 1960s onwards-both as a direct means of communications and as support for satellite applications such as navigation, remote sensing, etc. However, all communications links between satellites and ground stations must cross the ionosphere, and thus, the same as HF, may be subject to disruption by space weather effects in the ionosphere. This applies to all frequencies from very high frequency (VHF) to S-band, thus covering a wide range of satellite applications, today including L-band satcom, GNSS, etc. In addition, radio signals working at these frequencies are widely used for satellite tracking by ground-based radars and for active and passive observations of Earth's surface using satellite-based sensors.

\section{Where Are We Today?}

Today, there are continuing strong links between ionospheric science and the applications community. These include key physics challenges (a) to understand the spatial and temporal morphology of the ionosphere and thus how this slows and refracts radio signals as they pass through the ionosphere and (b) to understand the properties, and generation of, ionospheric irregularities that can diffract and scatter radio signals, leading to unexpected variations in the signal phase and amplitude. However, we must recognise that these challenges overlap with other challenges that arise from curiosity-driven research and from links with other scientific disciplines, such as:

1. The role of the high-latitude ionosphere as an image of magnetospheric dynamics-and hence the value of observations of that region as a means of studying magnetospheric dynamics. The high-latitude ionosphere is strongly coupled to the magnetosphere via the magnetic field lines that thread both regions, e.g., the flow of plasma in the high-latitude ionosphere is largely driven by magnetospheric electric fields, which are in turn driven by the flow of momentum from the solar wind into the magnetosphere. These flow patterns were first recognised over sixty years ago [26], and our understanding has gradually been refined over the years as advanced ionospheric instruments have been deployed in the Arctic and Antarctic (for example, see $[2,27,28]$ ) and as a range of space missions, such as Cluster and THEMIS, have provided new insights into magnetosphere-ionosphere coupling (for example, see $[29,30])$. However, we are still learning as there is much more to understand about the wide range of physics at work in the high-latitude ionosphere. Thus, new instruments are being developed such as the EISCAT_3D radar [31], which will enable simultaneous measurements across a large volume of the polar ionosphere. In addition, old techniques are being enhanced-such as the use of sounding rockets 
to observe the small-scale plasma physics (e.g., see [32]). Note that sounding rockets are vastly superior to satellites for this purpose as they can have a low velocity (100-200 $\mathrm{m} \mathrm{s}^{-1}$ ) across the magnetic field lines, and hence better resolution of plasma structures, compared to satellites, which must necessarily cross magnetic field lines much faster, typically $7 \mathrm{~km} \mathrm{~s}^{-1}$;

2. The weak coupling between the F-region ionosphere and the upper thermosphere. This is a critical scientific challenge as these two elements of the upper atmosphere co-exist in the same physical space, roughly $150 \mathrm{~km}$ to $500 \mathrm{~km}$ altitude. However, the thermosphere behaves as a fluid (as the mean free path of neutrals is less than a scale height), whilst the F-region ionosphere behaves as a collisionless plasma (as the ion-neutral collision frequency is less than the ion-gyrofrequency at these altitudes). The two elements are weakly coupled via ion-neutral collisions, which facilitate momentum transfers, leading to a variety of interesting effects including (a) vertical flows of F-region plasma driven by thermospheric winds at mid- and lowlatitudes (for example, see [33]) and (b) equatorward thermospheric winds around local midnight at high latitudes as a result of solar-wind-driven ionospheric flows from the dayside to the nightside (for example, see [34]). Many features arising from this coupling are understood, but there is a critical underlying issue that follows from the existence of such features. Namely, we cannot fully understand the behaviour of the ionosphere without also understanding the behaviour of the thermosphere, how it is driven by flows of energy and matter, both from above (e.g., extreme ultra-violet (EUV) radiation from the Sun, electric fields and currents from the magnetosphere) and from below (e.g., gravity waves from convective activity in the troposphere, upflows of molecular species from the mesosphere and lower thermosphere). These flows and their importance for observing and modelling the thermosphere remain an important area of study, for example as discussed by [35];

3. Ionospheres are ubiquitous across the universe. It is important to recognise that the co-existence of ionised (plasma) and neutral (fluid) components within the same atmosphere is a common feature of many astrophysical environments across the solar system and across the wider universe. Earth's upper atmosphere is simply the most accessible example of this type of environment and one where the presence of a significant magnetic field enriches the range of plasma behaviours in that environment. Thus, our understanding of Earth's ionosphere provides insights that can be of value for the fundamental understanding of partially ionised plasmas throughout the universe. A straightforward example has been the application of ionospheric expertise to improve our understanding of Mars' ionosphere. We now have a wealth of data from missions such as Mars Express and MAVEN and a good initial understanding of the ionosphere and other plasma environments of the Red Planet, enough to recognise that there is still much to learn [36]. However, this is just a second example (after Earth); there is considerable scope for novel research on ionospheres elsewhere in the solar system (around other planets, around comets, and in the solar chromosphere), as well as around exo-planets and other distant objects (e.g., accretion discs).

These are just three examples of how ionospheric science has links to other research disciplines and benefits from, and contributes to, curiosity-driven research. Other examples include wider links with magnetospheric physics (where we now appreciate that, but do not fully understand how, the mid- and low-latitude ionosphere is sometimes strongly influenced by magnetospheric electric fields) and with plasma physics (to study how plasma instabilities generate irregularities in many parts of the global ionosphere).

Nonetheless, we must recognise that ionospheric science has a strategic value that sits alongside the intellectual challenge that we expect in curiosity-driven research. In this respect, ionospheric science emulates many, perhaps all, environment sciences. A deep understanding of the environments that affect human activities enables us to exploit those environments for beneficial results and to protect ourselves against their natural adverse impacts (and to avoid creating further adverse impacts by degrading those environments). 
This is, of course, no more than applying science to live in harmony with the natural world-but that application is one of the great goals of science.

In the case of ionospheric science, strategic value comes primarily from insights into how ionospheric variations affect radio signals passing through that region, often for the worse, but sometimes for the better. We can apply our understanding to assess how and when ionospheric effects on radio signals will impact radio technologies. In addition, where that assessment reveals gaps in our knowledge, we can enhance that understanding through targeted research programmes, i.e., where funding agencies seek research proposals to address specific scientific goals already identified as strategically important by the agency. Such goals are typically set through consultation with a range of experts, including potential users of the research, as well as members of the research community. As a possible ionospheric example, targeted research can seek to improve our understanding of where and when ionospheric instabilities will occur with sufficient strength to distort the amplitude and phase of important radio signals (e.g., GNSS and satcom signals at L-band frequencies).

However, research outputs, whether from targeted or curiosity-driven research, are far from the end of the story. How do we transition research outcomes into operational use? This has traditionally been a significant problem, especially when transitioning research models into new services for forecasting and nowcasts. This is now being addressed through the development of "Research-to-Operations" (R20) programmes that bring researchers together with service providers to promote the development of high-quality modelling software and to support its validation in simulated operational environments. It is essential that such software be able to detect and smoothly deal with operational problems such as missing or poor-quality data, to alert operations teams to major problems, and to be secure against the hostile actions of those who might wish to disrupt operational services of all kinds. This need for dedicated actions to transition research into operations should also be complemented by feedback from operations teams to research teams. This "Operations-to-Research" (O2R) dialogue can be a great stimulus to researchers, triggering ideas for future research projects, both curiosity-driven and targeted. The support from operators can also help to build the case for funding that research. Thus, as discussed by Opgenoorth et al. [37], links between researchers and service operators can form an iterative loop (R2O2R) that can advance both high-quality science and high-quality services.

In summary, ionospheric science is now a mature field similar to many other environmental sciences. Whilst we have a good understanding of the basic science, there is a wealth of detail that needs to be explored and that has links to other disciplines including atmospheric science, plasma physics, magnetospheric physics, and planetary and exoplanetary science. There is much scope for curiosity-driven research as shown by a range of ionospheric effects that have been discovered over the past 20 years, e.g., the longitudinal modulation of the equatorial ionisation anomaly by energy flows from the troposphere (e.g., see [38]), the curious auroral structures known as STEVE (e.g., see [39]), and many others. However, as with other environmental sciences, there is also much scope for targeted research and to build strong links between researchers and service providers, both vital to focus research on strategic goals and then transition research outputs into operational use.

\section{Diversity Can Be a Challenge}

The diversity of funding approaches needed in ionospheric research can be an issue when ionospheric science is positioned alongside areas where the emphasis has traditionally been to support curiosity-driven research and, sometimes, to focus on the discovery of new science, rather than fully developing our physical understanding of natural environments. That full physical understanding is a major intellectual challenge wherever natural environments exhibit complexity, i.e., that environment is a system with many different elements, and the overall system behaviour is determined as much (if not mainly) by interactions between those elements, as by the physical properties of individual elements. Thus, a reductionistic approach to the study of the complex environments will inevitably fail, and 
a holistic approach is essential, as noted for space weather in general by Lugaz et al. [7]. This is certainly the case for the ionosphere, given its coupling not only to the co-located thermosphere, but also to the magnetosphere and to the lower atmosphere.

The experience of the author, and anecdotal reports from colleagues, suggests that the research challenge of fully understanding the behaviour of complex natural systems is not always understood by those working in funding systems focused mainly on curiositydriven research. In such a culture, the expansion of existing scientific knowledge that is essential to obtain full understanding may be assessed as an application of the existing knowledge and perhaps denied funding. While this would be a false assessment, it is important to recognise that it is a cultural problem. That the false assessment arises from a culture that does not grasp the diversity of approaches needed to fully develop scienceand hence that the application of the science, more accurately its transition to operational use, comes later. This is not at all to downplay the importance of curiosity-driven researchrather, it is to stress that it is part of a diverse culture of scientific research and that processes to determine funding need to recognise and reflect that diversity.

For ionospheric research, and indeed for many other aspects of research into space weather, one good way to approach this is to integrate ionospheric and other space weather research more closely with other environmental sciences. These sciences have a strong heritage of diverse research approaches-one that has evolved as our understanding of natural environments on Earth has become ever more important, both to make best use of the resources of our planet and to mitigate the risks posed by natural hazards. Modern ionospheric science fits perfectly in this framework: (a) it provides knowledge that helps us to transmit reliable radio signals across our planet and near-Earth space so as to support a wide range of societally vital applications (e.g., communications, navigation, timing services, remote sensing, etc.), and (b) it also helps us recognise and mitigate natural phenomena (e.g., space weather effects on the ionosphere) that can disrupt those applications.

This environmental science approach is also consistent with the growing development of many national and international space weather services that are embedded in the global network of meteorological services. That network already supports a range of services that monitor and forecast environmental conditions, obviously weather, but also other factors such as the atmospheric transport of volcanic ash away from eruptions. The latter provided a model for the establishment in 2019 of international space weather services for aviation under the auspices of the International Civil Aviation Organisation [40]. These services include information on ionospheric impacts to address aviation use of HF radio and GNSS services and were initially supported by three providers: the U.S. Space Weather Prediction Center [41], a consortium called PECASUS composed of European providers led by Finnish Meteorological Institute [42], and a consortium called ACFJ composed of providers based in Australia, Canada, France, and Japan [43]. They were joined in 2021 by a fourth provider in the form of a consortium between China and Russia [44], and it is expected that this support will soon expand to include a provision from South Africa. At a national level, there is also growing involvement of meteorological services in the national provision of space weather reports and forecasts, with examples including the USA, UK, Finland, and Australia.

Looking in detail, the UK provides an example of how ionospheric research can benefit from an environment science approach. Prior to 2008, research in solar-terrestrial physics, including studies of Earth's ionosphere, had long been funded as part of a wider astronomy and space programme that was created when the UK's Science Research Council (SRC, later SERC) was established in 1965 . That wider programme passed through many evolutions, for example with responsibility for space-based remote sensing of Earth being transferred to the UK's Natural Environment Research Council (NERC) in 1994. By 2008, the astronomy and space programme had become part of the UK's Science and Technology Facilities Council (STFC), which then proposed to cease supporting solar-terrestrial physics with respect to its manifestations on Earth, including studies of the Earth's ionosphere. This was 
part of a wide set of proposed changes to STFC programmes, many of which received a strong pushback from across the scientific community, even prompting significant debate in Parliament [45]. This pushback led to a review by an independent committee whose report included a recommendation that responsibility for solar-terrestrial physics be transferred to NERC [46]. This proposal was accepted by the Government, and that responsibility (and appropriate funding) was transferred to NERC in 2010. As that change has been implemented over the past decade, ionospheric researchers have had opportunities to bid for diverse forms of funding. Two recent major successes in this area are (a) NERC's role as the UK lead in the new EISCAT_3D incoherent scatter radar [47] and (b) NERC's support of targeted research to develop better ionospheric and thermospheric models $[48,49]$ and to transition those models into operational use at the UK Met Office [50]. The support from NERC has also included a range of smaller projects, with examples including preparatory research for scientific exploitation of EISCAT_3D [51,52] and translational research helping industry to assess ionospheric impacts on their use of GNSS [53]. In summary, UK researchers studying Earth's ionosphere (and other Earth-focused aspects of solar-terrestrial physics) are now increasingly linked to an environment science culture that is supportive of the diverse approaches needed to advance both the science and its application to real-world problems. It is a very welcome and positive change-and one that has fit well with the increasing attention given to space weather as part of the UK Government's policy, as shown by the publication in September 2021 of a new national strategy on preparedness for severe space weather conditions [54].

This increasing attention to space weather is mirrored in many other countries, as shown in surveys undertaken by the UN Expert Group on Space Weather. That group submitted a report in February 2022 [55], showing that, of approximately 40 countries that have provided national responses, the vast majority are either already supporting a range of space weather activities or are interested in working with other countries to develop those activities. These activities are not only research actions, but also observation and measurement programmes, risk and economic impact assessments, and the provision of space weather services. All these activities will necessarily include space weather effects in the ionosphere because those effects have profound impacts on radio technologies now vital to all countries around the world, developed and developing nations alike, because of their critical roles in transport and communications infrastructures.

This global concern about space weather suggests there is great value in encouraging countries to treat ionospheric science, indeed all scientific aspects of space weather, as part of the environmental sciences. That will help researchers to advance the science through a mix of curiosity-driven and targeted research and to build effective research-to-operations programmes (and also to facilitate feedback from operations to research). A survey of the current situation in different countries is beyond the scope of this paper. However, the author would be interested in learning more about those national situations.

\section{Summary}

Contemporary ionospheric research spans a spectrum of scientific activities starting with curiosity-driven research and ending with the application of research outcomes to practical problems. The former includes many research challenges such as the need to understand how Earth's ionosphere is coupled to other physical entities as discussed in previous sections. Today, these studies of Earth's ionosphere are complemented by studies of other ionospheres within the solar system, in particular Mars, for which we now have a wealth of ionospheric observations, and more generally studies of partially ionised plasmas across the universe. Earth's ionosphere is the most accessible example of this important class of astrophysical plasmas. The application of ionospheric science to practical problems focuses on radio signal propagation through the ionosphere. This is a wide field because of the great variety of systems that must consider ionospheric propagation and the range of frequencies that those systems use. These range at least from VLF systems working at frequencies of tens of $\mathrm{kHz}$, where signals propagate in the natural waveguide between the 
Earth's surface and ionosphere, up to S-band systems working at frequencies of $2-3 \mathrm{GHz}$, where ionospheric scintillation can distort signal propagation. A detailed discussion of those systems is well beyond the scope of this paper, so we just highlight their variety, and hence their sensitivity to a wide range of ionospheric features. Thus, the application of ionospheric science requires a wide and detailed understanding of the ionosphere.

These two boundary cases of curiosity-driven research and of applications of research outcomes are linked by two other research activities, namely targeted research and $\mathrm{R} 2 \mathrm{O} / \mathrm{O} 2 \mathrm{R}$ activities. In the case of targeted research, funding agencies define the research goals after a dialogue between scientists and potential users of research outcomes, but those goals still require cutting-edge research. This is a critical stage in refining research to the point where one can identify specific applications. The need for such targeted research is typical of a mature environment science; we know the basics, but not the details, and those details are crucial to the delivery of societal value from research. In the case of R20 and $\mathrm{O} 2 \mathrm{R}$, it is vital to establish, and fund, processes that facilitate interactions between researchers and service providers. Most obvious here is the need to facilitate the migration of forecast and nowcast models from research to operational environments, with all that implying high-quality management of software. The advent of cloud-based environments is providing a new and exciting basis for that migration to occur, not least of which because it strongly supports distributed working between science and operations teams based at different locations.

Thus, contemporary ionospheric research requires a diversity of approaches in a way that it is supported and carried out. Figure 1 provides an outline of how ideas might flow in that diverse culture, including the feedback that can drive a virtuous circle that can stimulate further progress in the science and its application. The need for diversity is increasingly recognised at an international level, e.g., through discussions on European [37] and global [56-59] coordination of space weather activities. Those reports also provide a call-to-arms for national efforts that can encourage and sustain those diverse approaches, whilst also stimulating, and benefiting from, improved international coordination and cooperation. The author encourages colleagues around the world to engage with these international efforts and, thus, advance our science.

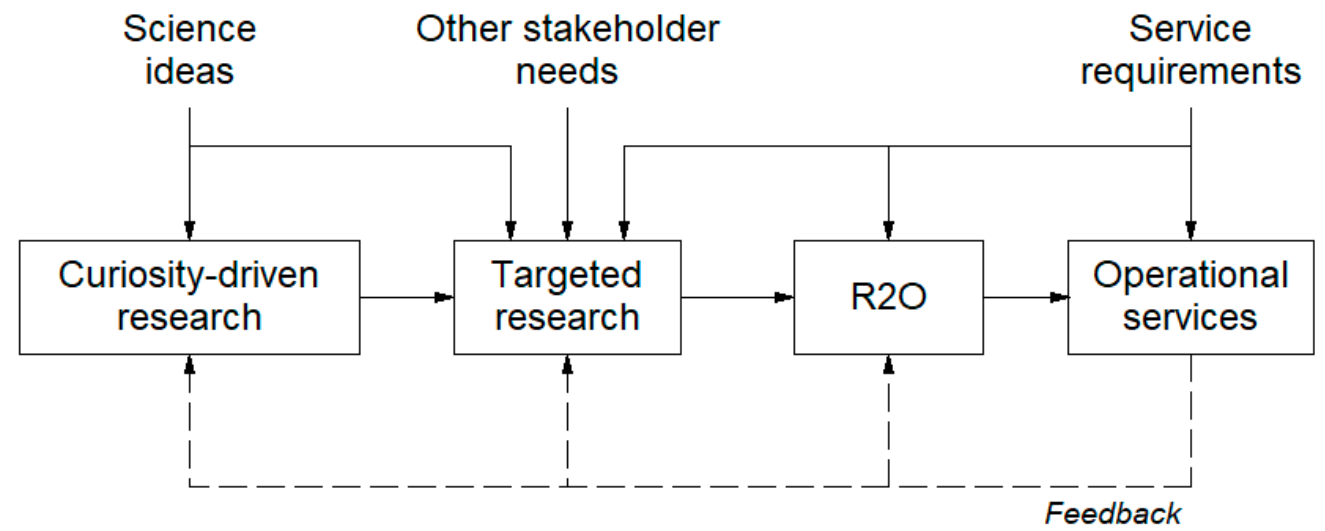

Figure 1. An outline of how ideas might flow in a diverse scientific culture as discussed in this paper. The research progresses, left to right, from curiosity-driven research to operational services, but the intermediate steps are vital and need dedicated processes to ensure that progress. Those processes must encourage scientists to engage not only with end users such as service operators, but also other stakeholders such as government agencies responsible for the regulation and resilience of services.

Funding: I acknowledge support provided by STFC, including partial support from Grant ST/ M001083/1.

Institutional Review Board Statement: Not applicable.

Informed Consent Statement: Not applicable. 
Data Availability Statement: This study did not generate any data.

Acknowledgments: This paper consolidates policy ideas that the author has developed over several decades of working with many colleagues across the scientific community, in particular the solarterrestrial physics community of which ionospheric physics is a key part, but also many interactions with colleagues across the wider astronomy and geophysics communities. The author is grateful for many stimulating, and sometimes challenging, discussions. However, the ideas presented here are solely the responsibility of the author.

Conflicts of Interest: The author declares no conflict of interest.

\section{References}

1. Rudaux, L.; de Vaucouleurs, G. Larousse Encyclopedia of Astronomy (English Edition); Hamlyn Publishing: Feltham, UK, 1959.

2. Rishbeth, H.; Van Eyken, A.P. EISCAT: Early history and the first ten years of operation. J. Atmos. Terr. Phys. 1993, 55, 525-542. [CrossRef]

3. Röttger, J.; Wannberg, U.G.; van Eyken, A.P. The EISCAT scientific association and the EISCAT Svalbard radar project. J. Geomag. Geoelec. 1995, 47, 669-679. [CrossRef]

4. Cannon, P.S.; Bradley, P.A. Ionospheric propagation. In Propagation of Radio Waves, 2nd ed.; Barclay, L.W., Ed.; Institute of Engineering and Technology: London, UK, 2003; pp. 313-334.

5. Cannon, P.S.; Angling, M.J.; Heaton, J.A.T.; Rogers, N.C.; Shukla, A.K. The effects of space weather on radio systems. In Effects of Space Weather on Technology Infrastructure; Daglis, I.A., Ed.; Springer: Dordrecht, The Netherlands, 2004; pp. $185-201$.

6. Gumbel, J.; Stegman, J. A tribute to Georg Witt. J. Atmos. Solar-Terr. Phys. 2015, 127, 3-7. [CrossRef]

7. Lugaz, N.; Gannon, J.; Hapgood, M.; Liu, H.; Paul O’Brien, T. Space weather as the nexus of applied and fundamental space science: The need for separate funding mechanisms and definition. Space Weather 2021, 19, e2020SW002695. [CrossRef]

8. Griffiths, H. Oliver Heaviside and the Heaviside layer. Phil. Trans. R. Soc. 2018, 376, 20170459. [CrossRef] [PubMed]

9. Appleton, E.; Barnett, M. Local Reflection of Wireless Waves from the Upper Atmosphere. Nature 1925, 115, 333-334. [CrossRef]

10. Breit, G.; Tuve, M. A Radio Method of Estimating the Height of the Conducting Layer. Nature 1925, 116, 357. [CrossRef]

11. Bates, D.R. Dissociative Recombination. Phys. Rev. 1950, 78, 492-493. [CrossRef]

12. Huurdeman, A.A. The Worldwide History of Telecommunications; Wiley-Blackwell: Hoboken, NJ, USA, 2003; ISBN 978-0471205050.

13. La Station Radio de Bordeaux-Lafayette. Raconte-Moi la Radio Web Site. Available online: http://leradiofil.com/lafayette.htm (accessed on 29 January 2022).

14. Anderson, C.N. Correlation of long wave transatlantic radio transmission with other factors affected by solar activity. Proc. Inst. Radio Eng. 1928, 16, 297-347. [CrossRef]

15. Marconi, S.G. Radio communication. Proc. Inst. Radio Eng. 1928, 16, 40-69. [CrossRef]

16. Dellinger, J.H. Sudden ionospheric disturbances. Terr. Magn. Atmos. Electr. 1937, 42, 49-53. [CrossRef]

17. Newton, H. An Active Group of Sunspots and Unusual Conditions in the Ionosphere. Nature 1936, 137, 363. [CrossRef]

18. Newton, H. Radio Fadings and Bright Solar Eruptions. Nature 1936, 138, 1017. [CrossRef]

19. Newton, H.W.; Barton, H.J. Bright Solar Eruptions and Radio Fadings During the Years 1935-1936. Mon. Not. Roy. Astro. Soc. 1937, 97, 594-611. [CrossRef]

20. Newton, H.W. Sunspots, bright eruptions and magnetic storms. Observatory 1939, 62, 318-326.

21. Newton, H.W. Geomagnetic "Crochet" Occurrence at Abinger, 1936-1946, and Allied Solar and Radio Data. Geophys. J. Int. 1948, 5, 200-215. [CrossRef]

22. Telephone File, The. UK Telephone History. Available online: https://www.britishtelephones.com/histuk.htm (accessed on 30 January 2022).

23. The Guardian. Radio Telephones out of Action, The Aurora Borealis, Railway Signals Upset. The Guardian, 27 January 1938 ; 14.

24. The Times. Curtain of Colour Across the Sky. The Times, 27 January 1938; 14.

25. Whatman, A.B. Observations made on the Ionosphere during Operations in Spitsbergen in 1942-1943. Proc. Phys. Society. Sect. B 1949, 62, 307. [CrossRef]

26. Axford, W.I.; Hines, C.O. A unifying theory of high-latitude geophysical phenomena and geomagnetic storms. Can. J. Phys. 1961, 39, 1433-1464. [CrossRef]

27. Greenwald, R.A.; Weiss, W.; Nielsen, E.; Thomson, N.R. STARE: A new radar auroral backscatter experiment in northern Scandinavia. Radio Sci. 1978, 13, 1021-1039. [CrossRef]

28. Chisham, G.; Lester, M.; Milan, S.E.; Freeman, M.P.; Bristow, W.A.; Grocott, A.; McWilliams, K.A.; Ruohoniemi, J.M.; Yeoman, T.K.; Dyson, P.L.; et al. A decade of the Super Dual Auroral Radar Network (SuperDARN): Scientific achievements, new techniques and future directions. Surv. Geophys. 2007, 28, 33-109. [CrossRef]

29. Case, N.A.; Grocott, A.; Fear, R.C.; Haaland, S.; Lane, J.H. Convection in the magnetosphere-ionosphere system: A multimission survey of its response to IMF By reversals. J. Geophys. Res. Sp. Phys. 2020, 125, e2019JA027541. [CrossRef]

30. Walsh, B.M.; Zou, Y. The role of magnetospheric plasma in solar wind-magnetosphere coupling: A review. J. Atmos. Solar-Terr. Phys. 2021, 219, 105644. [CrossRef] 
31. McCrea, I.; Aikio, A.; Alfonsi, L.; Belova, E.; Buchert, S.; Clilverd, M.; Engler, N.; Gustavsson, B.; Heinselman, C.; Kero, J.; et al. The science case for the EISCAT_3D radar. Prog. Earth Planet. Sci. 2015, 2, 21. [CrossRef]

32. Giono, G.; Ivchenko, N.; Sergienko, T.; Brändström, U. Multi-point measurements of the plasma properties inside an aurora from the SPIDER sounding rocket. J. Geophys. Res. Sp. Phys. 2021, 126, e2021JA029204. [CrossRef]

33. Kohl, H.; King, J.W. Atmospheric winds between 100 and $700 \mathrm{~km}$ and their effects on the ionosphere. J. Atmos. Terr. Phys. 1967, 29, 1045-1062. [CrossRef]

34. Aruliah, A.L.; Rees, D.; Fuller-Rowell, T.J. The combined effect of solar and geomagnetic activity on high latitude thermospheric neutral winds. Part I. Obs. J. Atmos. Terr. Phys. 1991, 53, 467-483. [CrossRef]

35. Heelis, R.A.; Maute, A. Challenges to understanding the Earth's ionosphere and thermosphere. J. Geophys. Res. Sp. Phys. 2020, 125, e2019JA027497. [CrossRef]

36. Sánchez-Cano, B.; Lester, M.; Andrews, D.J.; Opgenoorth, H.; Lillis, R.; Leblanc, F.; Fowler, C.M.; Fang, X.; Vaisberg, O.; Mayyasi, M.; et al. Mars' plasma system. Scientific potential of coordinated multipoint missions: "The next generation". Exp. Astron. 2021, 36, 1-36. [CrossRef]

37. Opgenoorth, H.J.; Wimmer-Schweingruber, R.F.; Belehaki, A.; Berghmans, D.; Hapgood, M.; Hesse, M.; Kauristie, K.; Lester, M.; Lilensten, J.; Messerotti, M.; et al. Assessment and recommendations for a consolidated European approach to space weather-as part of a global space weather effort. J. Space Weather. Space Clim. 2019, 9, A37. [CrossRef]

38. Immel, T.J.; Sagawa, E.; England, S.L.; Henderson, S.B.; Hagan, M.E.; Mende, S.B.; Frey, H.U.; Swenson, C.M.; Paxton, L.J. Control of equatorial ionospheric morphology by atmospheric tides. Geophys. Res. Lett. 2006, 33, L15108. [CrossRef]

39. MacDonald, E.A.; Donovan, E.; Nishimura, Y.; Case, N.A.; Gillies, D.M.; Gallardo-lacourt, B.; Archer, W.E.; Spanswick, E.L.; Bourassa, N.; Connors, M.; et al. New science in plain sight: Citizen scientists lead to the discovery of optical structure in the upper atmosphere. Sci. Adv. 2018, 4, 16-21. [CrossRef] [PubMed]

40. ICAO. New Global Aviation Space Weather Network Launched. Available online: https://www.icao.int/Newsroom/Pages/ New-global-aviation-space-weather-network-launched.aspx (accessed on 6 February 2022).

41. NOAA Space Weather Prediction Center. Available online: https://www.swpc.noaa.gov/communities/aviation-communitydashboard (accessed on 6 February 2022).

42. PECASUS for ICAO. Available online: https:/ / pecasus.eu (accessed on 6 February 2022).

43. Bureau of Meteorology, Space Weather Advisories. Available online: http://www.bom.gov.au/aviation/space-weatheradvisories (accessed on 6 February 2022).

44. Reuters. China Establishes Space Weather Centre with Russia-National Weather Service. Available online: https://www.reuters. $\mathrm{com} /$ business/environment/china-establishes-space-weather-centre-with-russia-national-weather-service-2021-11-17 (accessed on 6 February 2022).

45. House of Commons. Debate on 7 July 2008, Hansard Columns 1214-1219. Available online: https://publications.parliament.uk/ $\mathrm{pa} / \mathrm{cm} 200708 / \mathrm{cmhansrd} / \mathrm{cm} 080707 /$ debtext/80707-0014.htm (accessed on 6 February 2022).

46. RCUK (Research Councils UK). Review of UK Physics. Available online: https://webarchive.nationalarchives.gov.uk/ukgwa/20 110518094209/http://www.rcuk.ac.uk/reviews/current/physics/Pages/home.aspx (accessed on 6 February 2022).

47. BAS (British Antarctic Survey). Most Advanced Space Weather Radar to be Built in Arctic. Available online: https://www.bas.ac uk/media-post/most-advanced-space-weather-radar-to-be-built-in-arctic (accessed on 6 February 2022).

48. NERC. List of Awards for "Space Weather Instrumentation, Measurement, Modelling and Risk: Ionosphere (SWIMMR-I)". Available online: http:/ / gotw.nerc.ac.uk/list_split.asp?awardref=NE\%2FV002686\%2F1 (accessed on 6 February 2022).

49. NERC. List of Awards for "Space Weather Instrumentation, Measurement, Modelling and Risk: Thermosphere (SWIMMR-T)" Available online: http:/ / gotw.nerc.ac.uk/list_split.asp?awardref=NE\%2FV002708\%2F1 (accessed on 6 February 2022).

50. Met Office. Innovative Space Weather Monitoring Projects Receive UKRI Funding. Available online: https://blog.metoffice.gov. uk/2020/07/06/innovative-space-weather-monitoring-projects-receive-ukri-funding (accessed on 6 February 2022).

51. NERC. List of Awards for "EISCAT_3D: Fine-Scale Structuring, Scintillation, and Electrodynamics (FINESSE)". Available online: http:/ / gotw.nerc.ac.uk/list_split.asp?awardref=NE\%2FW003104\%2F1 (accessed on 6 February 2022).

52. NERC. List of Awards for "DRivers and Impacts of Ionospheric Variability with EISCAT-3D (DRIIVE)". Available online: http:/ / gotw.nerc.ac.uk/list_split.asp?awardref=NE\%2FW003325\%2F1 (accessed on 6 February 2022).

53. NERC. Award for "Space Weather Disruptions to Satellite Navigation and Telecommunications: Ionospheric Scintillation". Available online: http:/ / gotw.nerc.ac.uk/list_full.asp?pcode=NE\%2FR009082\%2F1 (accessed on 6 February 2022).

54. BEIS (Department for Business, Energy \& Industrial Strategy). UK Severe Space Weather Preparedness Strategy. Available online: https:/ / www.gov.uk/government/publications/uk-severe-space-weather-preparedness-strategy (accessed on 6 February 2022).

55. UNOOSA (United Nations Office for Outer Space Affairs). Non-Consensus Paper of the Expert Group on Space Weather on the Survey of the State of Member State Preparedness, and Current and Future Activities and Needs for Space Weather Impact Mitigation. Available online: https://www.unoosa.org/oosa/oosadoc/data/documents/2022/aac.105c.12022crp/aac.105c.12 022crp.10_0.html (accessed on 26 February 2022).

56. Schrijver, C.J.; Kauristie, K.; Aylward, A.D.; Denardini, C.M.; Gibson, S.E.; Glover, A.; Gopalswamy, N.; Grande, M.; Hapgood, M.; Heynderickx, D.; et al. Understanding space weather to shield society: A global road map for 2015-2025 commissioned by COSPAR and ILWS. Adv. Space Res. 2015, 55, 2745-2807. [CrossRef] 
57. UNOOSA (United Nations Office for Outer Space Affairs). Draft Final Report of the Expert Group on Space Weather: Towards Improved International Coordination for Space Weather Services. This Link Gives Access to Versions of the Report in All Five Official Languages of the United Nations. Available online: https://www.unoosa.org/oosa/oosadoc/data/documents/2022 /aac.105c.11/aac.105c.11.401_0.html (accessed on 26 February 2022).

58. COSPAR: International Space Weather Action Teams. Available online: https:/ /www.iswat-cospar.org (accessed on 11 February 2022).

59. World Meteorological Organisation-Space Weather Coordination. Available online: https://public.wmo.int/en/programmes/ wmo-space-programme/space-weather-coordination (accessed on 11 February 2022). 